\title{
Asymptotic optimality of SEPT in Bayesian scheduling
}

Citation for published version (APA):

Marban, S., Rutten, C., \& Vredeveld, T. (2010). Asymptotic optimality of SEPT in Bayesian scheduling. METEOR, Maastricht University School of Business and Economics. METEOR Research Memorandum No. 050 https://doi.org/10.26481/umamet.2010050

Document status and date:

Published: 01/01/2010

DOI:

10.26481/umamet.2010050

Document Version:

Publisher's PDF, also known as Version of record

\section{Please check the document version of this publication:}

- A submitted manuscript is the version of the article upon submission and before peer-review. There can be important differences between the submitted version and the official published version of record.

People interested in the research are advised to contact the author for the final version of the publication, or visit the DOI to the publisher's website.

- The final author version and the galley proof are versions of the publication after peer review.

- The final published version features the final layout of the paper including the volume, issue and page numbers.

Link to publication

\footnotetext{
General rights rights.

- You may freely distribute the URL identifying the publication in the public portal. please follow below link for the End User Agreement:

www.umlib.nl/taverne-license

Take down policy

If you believe that this document breaches copyright please contact us at:

repository@maastrichtuniversity.nl

providing details and we will investigate your claim.
}

Copyright and moral rights for the publications made accessible in the public portal are retained by the authors and/or other copyright owners and it is a condition of accessing publications that users recognise and abide by the legal requirements associated with these

- Users may download and print one copy of any publication from the public portal for the purpose of private study or research.

- You may not further distribute the material or use it for any profit-making activity or commercial gain

If the publication is distributed under the terms of Article $25 \mathrm{fa}$ of the Dutch Copyright Act, indicated by the "Taverne" license above, 


\section{Maastricht University}

Sebastián Marbán, Cyriel Rutten, Tjark Vredeveld

Asymptotic optimality of SEPT in Bayesian scheduling

$\mathrm{RM} / 10 / 050$

\section{METEOR}

Maastricht University School of Business and Economics

Maastricht Research Schocl of Economics

of Technology and Organization

PO. Box 616

NL- 6200 MD Mastricht

The Netherlands 


\title{
Asymptotic Optimality of SEPT in Bayesian Scheduling
}

\author{
Sebastián Marbán Cyriel Rutten Tjark Vredeveld \\ Department of Quantitative Economics, Maastricht University \\ P.O. Box 616, 6200 MD Maastricht, The Netherlands \\ Email: \{s.marban, c.rutten, t.vredeveld\}@maastrichtuniversity.nl
}

\begin{abstract}
We consider a stochastic scheduling problem in which there is uncertainty about parameters of the probability distribution of the processing times. We restrict ourselves to the setting in which there are two different classes of jobs. The processing times of the jobs are assumed to be exponentially distributed with parameters $\vartheta$ and $\mu$, depending on the class of the job. We consider a Bayesian framework in which $\mu$ is assumed to be known, whereas the value of $\vartheta$ is unknown. However, the scheduler has certain beliefs about this parameter and by processing jobs from this class, the scheduler can update his beliefs about $\vartheta$.

For the traditional stochastic scheduling variant, in which the parameters are known, of the problem under consideration, the policy that always processes a job with shortest expected processing time (SEPT) is an optimal policy. However, it has been shown that in the Bayesian framework, it is not an optimal policy. Therefore, we analyze the quality of SEPT. We show that SEPT is at most a factor $5 / 4$ worse than an optimal policy and that it is asymptotically optimal.
\end{abstract}

\section{Introduction}

Over the last few decades a vast amount of research has focused on stochastic scheduling problems, see, e. g., [17]. A full range of articles is concerned with criteria that guarantee the optimality of simple policies for special scheduling problems or the quality of non-optimal policies. All these papers have in common that the processing times of the jobs are random variables of which the parameters like expected value are known for certain. In this paper, we deviate from this assumption. That is, we study a stochastic scheduling problem in which also the parameters of the processing time distributions are uncertain. By adopting a Bayesian methodology, we can learn about these parameters by processing jobs and observing their processing times. We study a policy that is optimal for the classical stochastic scheduling problem, but is not necessarily optimal in the Bayesian framework. We show that this policy is only a constant factor away from optimal and is even asymptotically optimal.

Problem definition. Given is a set of jobs each of which needs to be scheduled on a single machine. This machine can process at most one job at a time and once a job has been started it must remain on the machine until completion, i. e., preemption of jobs is not allowed. Moreover, the machine and all jobs are available for processing from the beginning. The processing time of a job is a random variable. The goal is to minimize the total completion time, $\sum_{j} C_{j}$, in expectation. In traditional stochastic scheduling, we assume that the parameters of the processing time distributions are known. In this paper, we deviate from this assumption by assuming that these parameters are not known. However, we do have prior beliefs on their values which will be updated over time when new jobs are being processed.

The solution to a stochastic scheduling problem is not a simple schedule, but a so-called scheduling policy. We follow the notion of scheduling policies as proposed by Möhring, Radermacher, and 
Weiss $[14,15]$. Roughly spoken, a scheduling policy makes scheduling decisions at certain decision time points, and these decisions are based on information on the observed past, as well as the a priori knowledge of the input data of the problem. The policy, however, must not anticipate information about the future, such as the actual realizations of the processing times of jobs that have not yet been completed.

Whenever the parameters of the random variables are known, then an optimal policy for the problem under consideration is the Shortest Expected Processing Time policy (SEPT) [19]: as soon as the machine is idle, start processing the uncompleted job with shortest expected processing time. However, from [9] it follows that whenever these parameters are uncertain, SEPT is not necessarily optimal.

In this paper, we consider only two job classes $J^{A}$ and $J^{B}$, a setting that has been previously studied by Burnetas and Katehakis [1] and Hamada and Glazebrook [9]. Classes $J^{A}$ and $J^{B}$ consist of $n_{A}$ respectively $n_{B}$ independent jobs. The processing time of a job in class $J^{A}$ is a random variable, which is exponentially distributed with parameter $\vartheta$. Here, $\vartheta$ is assumed to be unknown but fixed. In this way, the problem distinguishes itself from traditional stochastic scheduling problems. The processing time of a job in class $J^{B}$ is a random variable which is exponentially distributed with known parameter $\mu$. Let $X$ and $Y$ represent two generic random variables for the processing times of a job of class $J^{A}$ and $J^{B}$, respectively. Note that $\mathbb{E}[Y]=\frac{1}{\mu}$. Furthermore, let random variable $X_{j}$ denote the processing time of the $j^{\text {th }}$ job of class $J^{A}$.

Bayesian methodology offers a method to formally recognize the uncertainty regarding $\vartheta$. For some $\theta>0$, let $g(\theta):=\operatorname{Pr}[\vartheta=\theta]$ denote a probability density function expressing how strongly we believe that the value of $\vartheta$ equals $\theta . g(\theta)$ is also referred to by prior, since it encapsulates all our knowledge regarding $\vartheta$ prior to seeing any realization of processing times of jobs of class $J^{A}$. In this paper, we assume $g(\theta)$ to be a gamma distribution with parameters $\omega>0$ and $\alpha>1$. Furthermore, let $g\left(\theta \mid x_{1}, \ldots, x_{n}\right):=\operatorname{Pr}\left[\vartheta=\theta \mid X_{1}=x_{1}, \ldots, X_{n}=x_{n}\right]$ be a probability density function expressing our beliefs regarding the value of $\vartheta$, once $n$ jobs of class $J^{A}$ have been completed with processing time realizations $x_{1}$ up to $x_{n} . g\left(\theta \mid x_{1}, \ldots, x_{n}\right)$ is a gamma distribution with parameters $\omega_{n}:=\omega+\sum_{i=1}^{n} x_{i}$ and $\alpha_{n}:=\alpha+n$, since the gamma and exponential are conjugate distributions. This result was already mentioned in Section 9.4 of [3] and is also trivially derived from Bayes' theorem for probability density functions.

These updates of beliefs regarding the unknown value of $\vartheta$ enable us to update our beliefs towards the processing time of the next job of class $J^{A}$ as well. The probability density function expressing these beliefs after having completed $n$ jobs of class $J^{A}$ is denoted by $f\left(x_{n+1}\right):=$ $\operatorname{Pr}\left[X_{n+1}=x_{n+1} \mid X_{1}=x_{1}, \ldots, X_{n}=x_{n}\right]$, which is equal to

$$
\begin{aligned}
f\left(x_{n+1}\right) & =\int_{0}^{\infty} f\left(x_{n+1} \mid \theta\right) g\left(\theta \mid x_{1}, \ldots, x_{n}\right) \partial \theta \\
& =\int_{0}^{\infty} \theta e^{-\theta x_{n+1}} \frac{\omega_{n}^{\alpha_{n}}}{\Gamma\left(\alpha_{n}\right)} \theta^{\alpha_{n}-1} e^{-\theta \omega_{n}} \partial \theta=\frac{\alpha_{n} \omega_{n}^{\alpha_{n}}}{\left(\omega_{n}+x_{n+1}\right)^{\alpha_{n}+1}} .
\end{aligned}
$$

Furthermore, straightforward integration yields the first moment of $X_{n+1}$

$$
\mathbb{E}\left[X_{n+1} \mid x_{1}, \ldots, x_{n}\right]=\int_{0}^{\infty} x_{n+1} f\left(x_{n+1}\right) \partial x_{n+1}=\frac{w_{n}}{\alpha_{n}-1} .
$$

In traditional stochastic scheduling an optimal policy is one that minimizes the total completion time in expectation. In the Bayesian framework, an optimal policy also minimizes the total completion time in expectation, but also underlies the uncertainty about the parameter $\vartheta$, i. e.,

$$
\mathbb{E}[\mathrm{OPT}]=\inf _{\Pi} \mathbb{E}_{g}\left[\sum_{j} C_{j}^{\Pi}\right],
$$

where $\mathbb{E}[\mathrm{OPT}]$ denotes the expected sum of completion times for the optimal policy OPT and $C_{j}^{\Pi}$ denotes the random variable of the completion time of job $j$ under policy $\Pi$. 
In terms of decision making, SEPT and OPT could be interpreted as having a short-term and long-term view, respectively. SEPT only processes a job of class $J^{A}$ in case the expected processing time of this job is minimal. OPT, however, might choose to process a job of class $J^{A}$ for which the expected processing time is not necessarily minimal. As a trade-off, OPT benefits from the additional information which is acquired regarding the uncertain parameter $\vartheta$. This information could then lead to better future decision making and a lower sum of expected completion times. From the above reasoning, one might expect SEPT to perform suboptimal. On the other hand, it is well-known that SEPT is optimal for stochastic single machine scheduling, see [19]. This raises the question how effective SEPT is within a Bayesian framework. A conclusive answer is presented in this paper.

Related work. Stochastic scheduling has been an active field of research for more than 40 years. In stochastic scheduling, the processing times of jobs are random variables for which the parameters of the underlying distribution are known. Rothkopf [19] shows that WSEPT (Weighted Shortest Expected Processing Time) is an optimal policy for the stochastic single machine scheduling problem, where the objective is to minimize the sum of weighted expected completion times in the absence of preemption. Sevcik [21] and Weiss [25] present an optimal policy for the same problem when preemption is allowed. Pinedo [16] considers the problem with due dates and release dates.

Weiss $[23,24]$ analyzes the performance of WSEPT for the stochastic parallel machine scheduling problem. His bounds yield asymptotic optimality of WSEPT for a certain class of processing time distributions. The first guarantee on the quality of an approximative policy was given by Möhring, Schulz, and Uetz [13]. Other approximative policies have been considered in $[22,11,20,12,2]$.

The scheduling problem studied in this paper is a Bayesian version of the problem discussed in [19]. To the best of our knowledge, there are only few other papers that also apply Bayesian methodology to stochastic scheduling problems. The pioneering paper of Gittins and Glazebrook [6] considers the problem of allocating processing time among jobs at every decision point. The processing time of each job is a random variable with probability density function depending on the same unknown parameter. The beliefs over this parameter are updated every time a job is completed or preempted. The optimal schedule is obtained by calculating appropriate dynamic allocation indices, which were first proposed by [7].

There are several other papers with results based on dynamic allocation indices. In Hamada and Glazebrook [9], optimal policies are obtained for a similar problem setting as discussed in this paper. They consider the problem with multiple job classes instead of two. Furthermore, they assign a weight to each job of a certain class. After formulating the problem as a dynamic program, optimal policies are derived using dynamic allocation indices which are similar to the ones constructed in $[4,5]$. Although they present the relevant indices for small instances, it is hard to retrieve them for large values of $\alpha$ or $n_{A}$, since the calculations require solving non-linear equations recursively.

Burnetas and Katehakis [1] also study a problem setting with two job classes, similar to the one discussed in this paper. Their analysis does neither limit the processing time of a job to be exponentially distributed nor the unknown parameter to be drawn from a gamma distribution. For this problem, they derive dynamic programming optimality conditions and show that an optimal policy does not depend on $n_{B}$. This simplifies the optimality conditions allowing for the problem to be modeled as a stopping problem. Thereafter, they obtain characterizations concerning the structure and properties of the optimal sequential allocation policies, for the case with processing times belonging to an exponential family with a single parameter. Finally, a policy is stated that approximates the decisions made by an optimal policy, in case of $n_{A}$ approaching infinity.

Rieder and Weishaupt [18] study the problem of a single server that has to schedule two classes of jobs with linear waiting costs. Probabilities are assigned to each job indicating whether the job leaves or stays in the system and thereby changes its class. A Bayesian approach is taken with respect to the unknown probabilities. The objective is to minimize the expected total cost over a finite planning horizon. 
Glazebrook and Owen [8] seek to quantify the difference between using scheduling policies based on Bayesian methodology and classical stochastic scheduling policies on a single machine. This difference is called the Value of an Adaptive Solution, because classical stochastic scheduling policies do not adapt their believes on the probability distributions. For a certain class of Bayesian scheduling problems, upper bounds on this difference are computed.

The first paper to provide an extension to multiple machines is Hamada and Tamaki [10]. They study the problem of assigning jobs of two classes, equivalent to those in this paper, to two identical machines. The problem is modeled as a dynamic program. For the special cases with either $n_{A}=1$ or $n_{B}=1$, explicit formulas for the sum of expected completion times are derived. Additionally, an optimal policy is claimed based on the ideas of dynamic allocation indices, for the case when $n_{A}=1$.

Our contribution. Whereas Glazebrook and Owen [8] try to bound the performance of policies that do not learn about jobs of class $J^{A}$ in terms of an optimal policy, we study the policy SEPT that adapts its beliefs about the mean processing time of class $J^{A}$ jobs. In this paper, we give a worst-case performance guarantee on the quality of SEPT. That is, we give an upper bound on the ratio $\mathbb{E}[\mathrm{SEPT}] / \mathbb{E}[\mathrm{OPT}]$, where $\mathbb{E}[\mathrm{SEPT}]$ and $\mathbb{E}[\mathrm{OPT}]$ denote the expected sum of completion times for SEPT and OPT. We will give two bounds on the performance guarantee of SEPT for the case that there is only one job in class $J^{B}$. This first bound shows that SEPT is asymptotically optimal, whereas the second bound gives a performance guarantee of $5 / 4$ also for few jobs of class $J^{A}$. Finally, we remark that due to Theorem 3.4 and Remark 3.5 of Burnetas and Katehakis [1] we conjecture that our results can be extended to the general case of arbitrary $n_{B} \geq 0$.

The remainder of this paper is organized as follows. In Section 2 , we discuss a dynamic program that provides an optimal policy from [9] as well as a few more preliminary results and notation. The asymptotical optimality of SEPT is shown in Section 3 and in Section 4 we give a better bound whenever there are only few class $J^{A}$ jobs.

\section{Dynamic programming formulation}

In this section, some results and notation are stated that are used throughout the remainder of the paper. First, we formulate this problem at hand as a dynamic program, adopting the notation of $[9]$.

Let $\left(n_{A}, n_{B}, \omega, \alpha\right) \in \mathbb{Z}_{+} \times \mathbb{Z}_{+} \times \mathbb{R}_{>0} \times \mathbb{R}_{>1}$ be a state vector encompassing all relevant information of the state the system is in. It consists of the number of jobs in class $J^{A}$ and $J^{B}$ as well as the parameters for the prior of the current belief for $\vartheta$. Note that we do not include the parameter $\mu$ of distribution for the jobs in class $J^{B}$ in this state as this parameter does not change during the process. If in state $\left(n_{A}, n_{B}, \omega, \alpha\right)$, a job of class $J^{A}$ is processed and completed having realization $x$, then the state changes to $\left(n_{A}-1, n_{B}, \omega+x, \alpha+1\right)$. On the other hand, if in state $\left(n_{A}, n_{B}, \omega, \alpha\right)$ a job of class $J^{B}$ is processed and completed, then the state changes to $\left(n_{A}, n_{B}-1, \omega, \alpha\right)$. Let $\mathbb{E}\left[\operatorname{SEPT}\left(n_{A}, n_{B}, \omega, \alpha\right)\right]$ and $\mathbb{E}\left[\mathrm{OPT}\left(n_{A}, n_{B}, \omega, \alpha\right)\right]$ be the expected sum of completion times when, respectively, SEPT and OPT are adopted from state $\left(n_{A}, n_{B}, \omega, \alpha\right)$ onwards. Further, let $F^{A}\left(n_{A}, n_{B}, \omega, \alpha\right)$ denote the sum of the expected completion times of a policy which first processes a job of class $J^{A}$ (assuming $n_{A} \geq 1$ ) and follows an optimal policy afterwards. Similarly for $F^{B}\left(n_{A}, n_{B}, \omega, \alpha\right)$.

Hamada and Glazebrook [9] gave the following dynamic program to compute the optimal policy.

$$
\mathbb{E}\left[\operatorname{OPT}\left(n_{A}, n_{B}, \omega, \alpha\right)\right]=\min \left\{F^{A}\left(n_{A}, n_{B}, \omega, \alpha\right) ; F^{B}\left(n_{A}, n_{B}, \omega, \alpha\right)\right\} \quad \forall n_{A}, n_{B} \geq 1
$$


with

$$
\begin{array}{ll}
\mathbb{E}\left[\operatorname{OPT}\left(n_{A}, 0, \omega, \alpha\right)\right]=F^{A}\left(n_{A}, 0, \omega, \alpha\right)=\frac{\omega}{\alpha-1} \sum_{i=1}^{n_{A}} i & \forall n_{A} \geq 0, \\
\mathbb{E}\left[\operatorname{OPT}\left(0, n_{B}, \omega, \alpha\right)\right]=F^{B}\left(0, n_{B}, \omega, \alpha\right)=\frac{1}{\mu} \sum_{i=1}^{n_{B}} i & \forall n_{B} \geq 0 .
\end{array}
$$

As the length of the first job to be processed by a policy influences the completion time of all jobs, straightforward calculations show that

$F^{A}\left(n_{A}, n_{B}, \omega, \alpha\right)=\left(n_{A}+n_{B}\right) \frac{\omega}{\alpha-1}+\int_{x} \mathbb{E}\left[\mathrm{OPT}\left(n_{A}-1, n_{B}, \omega+x, \alpha+1\right)\right] f(x) \partial x \quad \forall n_{A} \geq 1$,

$F^{B}\left(n_{A}, n_{B}, \omega, \alpha\right)=\left(n_{A}+n_{B}\right) \frac{1}{\mu}+\mathbb{E}\left[\operatorname{OPT}\left(n_{A}, n_{B}-1, \omega, \alpha\right)\right] \quad \forall n_{B} \geq 1$,

where $f(x)$ is the probability density function of the processing time of a job of class $J^{A}$.

In the following lemma, we show that if SEPT starts processing a job of class $J^{A}$, then OPT will do so too.

Lemma 1. Suppose the system is in state $\left(n_{A}, 1, \omega, \alpha\right)$. If SEPT starts processing a job of class $J^{A}$, then OPT starts processing a job of class $J^{A}$.

Proof. By the dynamic programming formulation, OPT starts processing a job of class $J^{A}$ if $F^{A}\left(n_{A}, 1, \omega, \alpha\right) \leq F^{B}\left(n_{A}, 1, \omega, \alpha\right)$. SEPT starts processing a job of class $J^{A}$, so $\frac{1}{\mu} \leq \frac{\omega}{\alpha-1}$. We have,

$$
F^{B}\left(n_{A}, 1, \omega, \alpha\right)=\frac{n_{A}+1}{\mu}+\mathbb{E}\left[\operatorname{OPT}\left(n_{A}, 0, \omega, \alpha\right)\right]=\frac{n_{A}+1}{\mu}+\frac{\omega}{\alpha-1} \sum_{i=1}^{n_{A}} i .
$$

Further,

$$
\begin{aligned}
F^{A}\left(n_{A}, 1, \omega, \alpha\right) & =\left(n_{A}+1\right) \frac{\omega}{\alpha-1}+\int_{x} \mathbb{E}\left[\operatorname{OPT}\left(n_{A}-1,1, \omega+x, \alpha+1\right)\right] f(x) \partial x \\
& \leq\left(n_{A}+1\right) \frac{\omega}{\alpha-1}+\int_{x} F^{B}\left(n_{A}-1,1, \omega+x, \alpha+1\right) f(x) \partial x \\
& =\left(n_{A}+1\right) \frac{\omega}{\alpha-1}+\int_{x}\left(\frac{n_{A}}{\mu}+\frac{\omega+x}{\alpha} \sum_{i=1}^{n_{A}-1} i\right) f(x) \partial x=\frac{n_{A}}{\mu}+\frac{\omega}{\alpha-1}\left(1+\sum_{i=1}^{n_{A}} i\right) .
\end{aligned}
$$

Combining both expressions, we find

$$
\begin{aligned}
F^{A}\left(n_{A}, 1, \omega, \alpha\right) & -F^{B}\left(n_{A}, 1, \omega, \alpha\right) \\
& \leq\left(\frac{n_{A}}{\mu}+\frac{\omega}{\alpha-1}\left(1+\sum_{i=1}^{n_{A}} i\right)\right)-\left(\frac{n_{A}+1}{\mu}+\frac{\omega}{\alpha-1} \sum_{i=1}^{n_{A}} i\right)=\frac{\omega}{\alpha-1}-\frac{1}{\mu} \leq 0 .
\end{aligned}
$$

\section{Asymptotic optimality of SEPT}

In this section, we show that whenever the number of jobs of class $J^{A}$ tends to infinity, the ratio $\mathbb{E}[\mathrm{SEPT}] / \mathbb{E}[\mathrm{OPT}]$ tends to 1 . Before we prove this, we first provide the following lemmas.

Lemma 2. For $n_{A} \geq 2$,

$$
\int_{x} \mathbb{E}\left[\operatorname{OPT}\left(n_{A}, 1, \omega+x, \alpha+1\right)\right] f(x) \partial x>\frac{1}{\mu}+\frac{\omega}{\alpha-1} \sum_{i=1}^{n_{A}} i .
$$


Proof. First, we prove the statement for $n_{A}=2$.

$$
\begin{array}{rl}
\int_{x} & \mathbb{E} \\
& {[\operatorname{OPT}(2,1, \omega+x, \alpha+1)] f(x) \partial x} \\
& =\int_{x} \min \left\{3 \frac{1}{\mu}+3 \frac{\omega+x}{\alpha} ; 3 \frac{\omega+x}{\alpha}+\int_{x_{2}} \mathbb{E}\left[\operatorname{OPT}\left(1,1, \omega+x+x_{2}, \alpha+2\right)\right] f\left(x_{2}\right) \partial x_{2}\right\} f(x) \partial x \\
& >\int_{x}\left(\frac{1}{\mu}+3 \frac{\omega+x}{\alpha}\right) f(x) \partial x=\frac{1}{\mu}+3 \frac{\omega}{\alpha-1}=\frac{1}{\mu}+\frac{\omega}{\alpha-1} \sum_{i=1}^{2} i .
\end{array}
$$

Next, assume that, given some $k \geq 2$, the statement is correct for all $n_{A} \leq k$. We will show that it is also valid for $n_{A}=k+1$.

$$
\begin{aligned}
\int_{x} \mathbb{E} & {[\mathrm{OPT}(k+1,1, \omega+x, \alpha+1)] f(x) \partial x } \\
& =\int_{x} \min \left\{\frac{k+2}{\mu}+\frac{\omega+x}{\alpha} \sum_{i=1}^{k+1} i ;(k+2) \frac{\omega+x}{\alpha}+\int_{x_{2}} \mathbb{E}\left[\mathrm{OPT}\left(k, 1, \omega+x+x_{2}, \alpha+2\right)\right] f\left(x_{2}\right) \partial x_{2}\right\} f(x) \partial x \\
& >\int_{x} \min \left(\frac{k+2}{\mu}+\frac{\omega+x}{\alpha} \sum_{i=1}^{k+1} i ;(k+2) \frac{\omega+x}{\alpha}+\frac{1}{\mu}+\frac{\omega+x}{\alpha} \sum_{i=1}^{k} i\right) f(x) \partial x \\
& >\int_{x}\left\{\frac{1}{\mu}+\frac{\omega+x}{\alpha} \sum_{i=1}^{k+1} i\right\} f(x) \partial x=\frac{1}{\mu}+\frac{\omega}{\alpha-1} \sum_{i=1}^{k+1} i
\end{aligned}
$$

where the first inequality follows from the induction step.

Lemma 3. For all $n_{A} \geq 3$, if $F^{A}\left(n_{A}, 1, \omega, \alpha\right) \leq F^{B}\left(n_{A}, 1, \omega, \alpha\right)$, then

$$
\mathbb{E}\left[\operatorname{OPT}\left(n_{A}, 1, \omega, \alpha\right)\right]>\frac{1}{\mu}+\frac{\omega}{\alpha-1}\left(1+\sum_{i=1}^{n_{A}} i\right)
$$

Proof. If $F^{A}\left(n_{A}, 1, \omega, \alpha\right) \leq F^{B}\left(n_{A}, 1, \omega, \alpha\right)$, then

$$
\begin{aligned}
\mathbb{E}\left[\mathrm{OPT}\left(n_{A}, 1, \omega, \alpha\right)\right] & =F^{A}\left(n_{A}, 1, \omega, \alpha\right) \\
& =\left(n_{A}+1\right) \frac{\omega}{\alpha-1}+\int_{x} \mathbb{E}\left[\operatorname{OPT}\left(n_{A}-1,1, \omega+x, \alpha+1\right)\right] f(x) \partial x \\
& >\left(n_{A}+1\right) \frac{\omega}{\alpha-1}+\frac{1}{\mu}+\frac{\omega}{\alpha-1} \sum_{i=1}^{n_{A}-1} i=\frac{1}{\mu}+\frac{\omega}{\alpha-1}\left(1+\sum_{i=1}^{n_{A}} i\right),
\end{aligned}
$$

where the inequality follows from Lemma 2 .

Lemma 4. For all $n_{A} \geq 1$,

$$
\mathbb{E}\left[\operatorname{SEPT}\left(n_{A}, 1, \omega, \alpha\right)\right]<\frac{1}{\mu}+\frac{\omega}{\alpha-1} \sum_{i=1}^{n_{A}+1} i .
$$

Proof. First, we prove the statement for $n_{A}=1$. If $\frac{1}{\mu} \leq \frac{\omega}{\alpha-1}$, then $\mathbb{E}[\operatorname{SEPT}(1,1, \omega, \alpha)]=\frac{2}{\mu}+$ $\frac{\omega}{\alpha-1} \leq \frac{1}{\mu}+2 \frac{\omega}{\alpha-1}$. Otherwise, $\mathbb{E}[\operatorname{SEPT}(1,1, \omega, \alpha)]=2 \frac{\omega}{\alpha-1}+\frac{1}{\mu}$. Therefore,

$$
\mathbb{E}[\operatorname{SEPT}(1,1, \omega, \alpha)] \leq \frac{1}{\mu}+2 \frac{\omega}{\alpha-1}<\frac{1}{\mu}+\frac{\omega}{\alpha-1} \sum_{i=1}^{2} i .
$$


Next, assume that, given some $k \geq 1$, the statement holds for all $n_{A} \leq k$. We will show it also holds for $n_{A}=k+1$. If $\frac{1}{\mu} \leq \frac{\omega}{\alpha-1}$, then

$$
\mathbb{E}[\operatorname{SEPT}(k+1,1, \omega, \alpha)]=\frac{k+2}{\mu}+\frac{\omega}{\alpha-1} \sum_{i=1}^{k+1} i<\frac{1}{\mu}+\frac{\omega}{\alpha-1} \sum_{i=1}^{k+2} i .
$$

Otherwise,

$$
\begin{aligned}
\mathbb{E}[\operatorname{SEPT}(k+1,1, \omega, \alpha)] & =(k+2) \frac{\omega}{\alpha-1}+\int_{x} \mathbb{E}[\operatorname{SEPT}(k, 1, \omega+x, \alpha+1)] f(x) \partial x \\
& <(k+2) \frac{\omega}{\alpha-1}+\int_{x}\left(\frac{1}{\mu}+\frac{\omega+x}{\alpha} \sum_{i=1}^{k+1} i\right) f(x) \partial x=\frac{1}{\mu}+\frac{\omega}{\alpha-1} \sum_{i=1}^{k+2} i,
\end{aligned}
$$

where the inequality follows from the induction step. Hence,

$$
\mathbb{E}[\operatorname{SEPT}(k+1,1, \omega, \alpha)]<\frac{1}{\mu}+\frac{\omega}{\alpha-1} \sum_{i=1}^{k+2} i .
$$

Using these lemmas, we can prove the following theorem

Theorem 1. For all $n_{A} \geq 3$,

$$
\mathbb{E}\left[\operatorname{SEPT}\left(n_{A}, 1, \omega, \alpha\right)\right]<\frac{n_{A}+3}{n_{A}+1} \mathbb{E}\left[\mathrm{OPT}\left(n_{A}, 1, \omega, \alpha\right)\right] .
$$

Proof. First, consider the case where $F^{B}\left(n_{A}, 1, \omega, \alpha\right)<F^{A}\left(n_{A}, 1, \omega, \alpha\right)$. From Lemma 1, we know that it cannot occur that OPT starts processing a job of class $J^{B}$, while SEPT starts processing a job of class $J^{A}$. Therefore, if OPT starts processing a job of class $J^{B}$, then SEPT will do so too. Since there is only a single job of class $J^{B}$, SEPT and OPT yield the same schedule and it follows that $\mathbb{E}\left[\operatorname{SEPT}\left(n_{A}, 1, \omega, \alpha\right)\right]=\mathbb{E}\left[\operatorname{OPT}\left(n_{A}, 1, \omega, \alpha\right)\right]$.

Next, consider the case $F^{A}\left(n_{A}, 1, \omega, \alpha\right) \leq F^{B}\left(n_{A}, 1, \omega, \alpha\right)$. Then, by Lemmas 3 and 4 , we can bound the value of SEPT by

$$
\begin{aligned}
\mathbb{E}\left[\operatorname{SEPT}\left(n_{A}, 1, \omega, \alpha\right)\right] & <\frac{1}{\mu}+\frac{\omega}{\alpha-1} \sum_{i=1}^{n_{A}+1} i \\
& <\mathbb{E}\left[\operatorname{OPT}\left(n_{A}, 1, \omega, \alpha\right)\right]+n_{A} \frac{\omega}{\alpha-1} \\
& <\mathbb{E}\left[\operatorname{OPT}\left(n_{A}, 1, \omega, \alpha\right)\right]+n_{A} \frac{\mathbb{E}\left[\mathrm{OPT}\left(n_{A}, 1, \omega, \alpha\right)\right]}{\sum_{i=1}^{n_{A}} i} \\
& <\left(1+\frac{2}{n_{A}+1}\right) \mathbb{E}\left[\mathrm{OPT}\left(n_{A}, 1, \omega, \alpha\right)\right]=\frac{n_{A}+3}{n_{A}+1} \mathbb{E}\left[\mathrm{OPT}\left(n_{A}, 1, \omega, \alpha\right)\right],
\end{aligned}
$$

where the third inequality follows from Lemma 3 , since

$$
\mathbb{E}\left[\operatorname{OPT}\left(n_{A}, 1, \omega, \alpha\right)\right]>\frac{1}{\mu}+\frac{\omega}{\alpha-1}\left(1+\sum_{i=1}^{n_{A}} i\right)>\frac{\omega}{\alpha-1} \sum_{i=1}^{n_{A}} i .
$$

\section{Corollary 1.}

$$
\lim _{n_{A} \rightarrow \infty} \frac{\mathbb{E}\left[\operatorname{SEPT}\left(n_{A}, 1, \omega, \alpha\right)\right]}{\mathbb{E}\left[\operatorname{OPT}\left(n_{A}, 1, \omega, \alpha\right)\right]}=1
$$




\section{Performance guarantee for SEPT}

From Theorem 1 it follows that if there are only three jobs of class $J^{A}$, the performance guarantee is bounded by $3 / 2$. Similarly, Theorem 1 yields a performance guarantee exceeding $5 / 4$ if the number of jobs of class $J^{A}$ is less than seven. In this section, we show an improved performance guarantee of SEPT which is at most $5 / 4$, for any arbitrary number of jobs of class $J^{A}$ and a single job of class $J^{B}$.

\subsection{Performance guarantee when in state $(2,1, \omega, \alpha)$}

In this subsection, we provide a performance guarantee of $6 / 5$ for SEPT when there are only two jobs of class $J^{A}$. First, we state some preliminary results.

\section{Lemma 5.}

$$
\begin{aligned}
& F^{A}(2,1, \omega, \alpha)= \begin{cases}\frac{5 \omega}{\alpha-1}-\left(\frac{\mu \omega}{\alpha}\right)^{\alpha} \frac{1}{\mu(\alpha-1)}+\frac{1}{\mu} & \text { if } \alpha \geq \omega \mu \\
\frac{4 \omega}{\alpha-1}+\frac{2}{\mu} & \text { if } \alpha<\omega \mu\end{cases} \\
& F^{B}(2,1, \omega, \alpha)=\frac{3}{\mu}+\frac{3 \omega}{\alpha-1}
\end{aligned}
$$

Proof. The value for $F^{B}(2,1, \omega, \alpha)$ follows immediately from the fact that once the single job of class $J^{B}$ has been processed, only jobs of class $J^{A}$ are left to be processed.

To see the value for $F^{A}(2,1, \omega, \alpha)$, recall that the probability density function of the processing time of a job of class $J^{A}$ is $f(x):=\frac{\alpha \omega^{\alpha}}{(\omega+x)^{\alpha+1}}$. Then,

$$
F^{A}(2,1, \omega, \alpha)=3 \frac{\omega}{\alpha-1}+\int_{0}^{\infty} \min \left\{\frac{2}{\mu}+\frac{\omega+x}{\alpha} ; \frac{1}{\mu}+2 \frac{\omega+x}{\alpha}\right\} f(x) \partial x
$$

We distinguish between two cases. If $\alpha \leq \omega \mu$, then

$$
F^{A}(2,1, \omega, \alpha)=3 \frac{\omega}{\alpha-1}+\int_{0}^{\infty}\left\{\frac{2}{\mu}+\frac{\omega+x}{\alpha}\right\} f(x) \partial x=\frac{2}{\mu}+4 \frac{\omega+x}{\alpha} .
$$

Next, if $\alpha>\omega \mu$, then

$$
\begin{aligned}
F^{A}(2,1, \omega, \alpha) & =3 \frac{\omega}{\alpha-1}+\int_{0}^{\frac{\alpha}{\mu}-\omega}\left\{\frac{1}{\mu}+2 \frac{\omega+x}{\alpha}\right\} f(x) \partial x+\int_{\frac{\alpha}{\mu}-\omega}^{\infty}\left\{\frac{2}{\mu}+\frac{\omega+x}{\alpha}\right\} f(x) \partial x \\
& =\frac{5 \omega}{\alpha-1}+\frac{1}{\mu}-\frac{1}{\mu(\alpha-1)}\left(\frac{\mu \omega}{\alpha}\right)^{\alpha},
\end{aligned}
$$

where the last equality follows straightforwardly from integration and rearrangement of the terms.

Lemma 6. If the first job to be processed by SEPT is of the same class as the first job to be processed by $\mathrm{OPT}$, then $\mathbb{E}[\operatorname{SEPT}(2,1, \omega, \alpha)]=\mathbb{E}[\mathrm{OPT}(2,1, \omega, \alpha)]$.

Proof. If both SEPT and OPT start processing the job of class $J^{B}$, then after finishing this job, only jobs of class $J^{A}$ are left to be processed. Therefore, both OPT and SEPT behave the same.

On the other hand, if both SEPT and OPT start processing a job of class $J^{A}$, then after completion of this job, there is one job of class $J^{A}$ and one job of class $J^{B}$ left to be processed. Processing this single job of class $J^{A}$ reveals information regarding the unknown parameter $\vartheta$. However, as afterwards no more jobs of class $J^{A}$ are to be processed, this additional piece of information is of no value. Hence, OPT will follow SEPT. 
The lemma above implies that when both SEPT and OPT start processing a job of the same class, the performance guarantee equals 1 . Consequently, and due to Lemma 1, we can hereafter focus on the case where SEPT starts processing a job of class $J^{B}$, whereas OPT starts processing a job of class $J^{A}$. Then, $\frac{1}{\mu} \leq \frac{\omega}{\alpha-1}$ and $F^{A}(2,1, \omega, \alpha)<F^{B}(2,1, \omega, \alpha)$. The following lemma shows that when these inequalities are valid, also $\alpha>\omega \mu$ needs to hold.

Lemma 7. If $\frac{1}{\mu} \leq \frac{\omega}{\alpha-1}$ and $F^{A}(2,1, \omega, \alpha)<F^{B}(2,1, \omega, \alpha)$, then $\alpha>\omega \mu$.

Proof. We provide a proof by contradiction. Assume that $\alpha<\omega \mu$. Then, $\frac{1}{\mu}<\frac{\omega}{\alpha}<\frac{\omega}{\alpha-1}$. Consequently, $F^{B}(2,1, \omega, \alpha)=\frac{3}{\mu}+\frac{3 \omega}{\alpha-1}<\frac{2}{\mu}+\frac{4 \omega}{\alpha-1}=F^{A}(2,1, \omega, \alpha)$, yielding a contradiction.

To prove the bound on the performance guarantee for the state $(2,1, \omega, \alpha)$, we need to compute the limit in the following proposition.

\section{Proposition 1.}

$$
\lim _{\beta \downarrow 1} \frac{1}{\beta}\left(\frac{\beta-1}{\beta}\right)^{\beta-1}=1
$$

Proof. Let $v(\beta)=\beta \ln \beta=\ln \beta /(1 / \beta)$. It follows trivially from l'Hôpital's rule that

$$
\lim _{\beta \downarrow 0} v(\beta)=0 .
$$

Further, let $w(\beta)=\frac{1}{\beta}\left(\frac{\beta-1}{\beta}\right)^{\beta-1}$. Then,

$$
\begin{aligned}
\ln w(\beta) & =\ln \frac{1}{\beta}\left(\frac{\beta-1}{\beta}\right)^{\beta-1}=\ln \frac{(\beta-1)^{\beta-1}}{\beta^{\beta}} \\
& =(\beta-1) \ln (\beta-1)-\beta \ln \beta
\end{aligned}
$$

and

$$
\lim _{\beta \downarrow 1} \ln w(\beta)=\lim _{\beta \downarrow 1}\{(\beta-1) \ln (\beta-1)-\beta \ln \beta\}=0,
$$

where the last equality follows from (1). Since $u=\exp (\ln u)$, this brings us to

$$
\lim _{\beta \downarrow 1} w(\beta)=\lim _{\beta \downarrow 1} \exp (\ln w(\beta))=\exp (0)=1,
$$

which concludes the proof.

Theorem 2.

$$
\mathbb{E}[\operatorname{SEPT}(2,1, \omega, \alpha)]<\frac{6}{5} \mathbb{E}[\operatorname{OPT}(2,1, \omega, \alpha)]
$$

Proof. By Lemmas 1 and 6, we only need to consider the case in which SEPT starts processing a job of class $J^{B}$, whereas OPT first processes a job of class $J^{A}$. By Lemma 7 , we know that in this case $\alpha \geq \omega \mu$. Moreover, as the first job to be processed by SEPT is a job of class $J^{B}$, we also have that $\frac{1}{\mu} \leq \frac{\omega}{\alpha-1}$. Hence, $\alpha-1 \leq \omega \mu \leq \alpha$. Furthermore, it follows from Lemma 5 that

$$
\begin{aligned}
\mathbb{E}[\operatorname{SEPT}(2,1, \omega, \alpha)] & =\frac{3}{\mu}+\frac{3 \omega}{\alpha-1}, \\
\mathbb{E}[\operatorname{OPT}(2,1, \omega, \alpha)] & =\frac{5 \omega}{\alpha-1}-\left(\frac{\omega \mu}{\alpha}\right)^{\alpha} \frac{1}{\mu(\alpha-1)}+\frac{1}{\mu} .
\end{aligned}
$$


Let $\xi$ be defined by $\xi:=\omega \mu-(\alpha-1)$. From the above considerations, we know that $\xi \in[0,1]$. The difference between the sums of expected completion times of SEPT and OPT can be written in terms of $\alpha$ and $\xi$ as follows

$$
\begin{aligned}
\Delta(\alpha, \xi) & =\mathbb{E}[\operatorname{SEPT}(2,1, \omega, \alpha)]-\mathbb{E}[\operatorname{OPT}(2,1, \omega, \alpha)]=\frac{2}{\mu}-\frac{2 \omega}{\alpha-1}+\left(\frac{\omega \mu}{\alpha}\right)^{\alpha} \frac{1}{\mu(\alpha-1)} \\
& =\frac{2}{\mu}-\frac{2(\xi+\alpha-1)}{\mu(\alpha-1)}+\left(\frac{\xi+\alpha-1}{\alpha}\right)^{\alpha} \frac{1}{\mu(\alpha-1)} .
\end{aligned}
$$

Standard calculus techniques show that $\Delta(\alpha, \xi)$ is decreasing in $\xi$ on the interval $\xi \in[0,1]$ for any $\alpha>1$ and any $\mu>0$. Moreover, it can be shown that $\Delta(\alpha, 0)$ is decreasing in $\alpha$ for $\alpha>1$. Therefore, we can bound (2) by

$$
\Delta(\alpha, \xi) \leq \Delta(\alpha, 0) \leq \lim _{\alpha \downarrow 1} \Delta(\alpha, 0)=\lim _{\alpha \downarrow 1} \frac{1}{\mu(\alpha-1)}\left(\frac{\alpha-1}{\alpha}\right)^{\alpha}=\frac{1}{\mu},
$$

by Proposition 1.

Next, we rewrite the sum of expected completion times of OPT in terms of $\alpha$ and $\xi$

$$
\begin{aligned}
\mathbb{E}[\operatorname{OPT}(2,1, \omega, \alpha)] & =\frac{5 \omega}{\alpha-1}-\left(\frac{\omega \mu}{\alpha}\right)^{\alpha} \frac{1}{\mu(\alpha-1)}+\frac{1}{\mu} \\
& =\frac{5(\xi+\alpha-1)}{\mu(\alpha-1)}-\left(\frac{\xi+\alpha-1}{\alpha}\right)^{\alpha} \frac{1}{\mu(\alpha-1)}+\frac{1}{\mu} .
\end{aligned}
$$

Standard calculus techniques show that this value is increasing in $\xi$ on $\xi \in[0,1]$. Therefore,

$$
\mathbb{E}[\operatorname{OPT}(2,1, \omega, \alpha)]>\frac{6}{\mu}-\left(\frac{\alpha-1}{\alpha}\right)^{\alpha} \frac{1}{\mu(\alpha-1)}>\frac{5}{\mu},
$$

where the last inequality is due to the fact that $\left(\frac{\alpha-1}{\alpha}\right)^{\alpha} \frac{1}{\alpha-1}$ is decreasing in $\alpha$ for $\alpha>1$ and, by Proposition 1, we know that this value tends to 1 when $\alpha$ tends to 1 .

Finally, combining (2), (3), and (4) yields the desired result

$$
\begin{aligned}
\mathbb{E}[\operatorname{SEPT}(2,1, \omega, \alpha)] & =\mathbb{E}[\operatorname{OPT}(2,1, \omega, \alpha)]+\Delta(\alpha, \xi) \leq \mathbb{E}[\operatorname{OPT}(2,1, \omega, \alpha)]+\frac{1}{\mu} \\
& <\frac{6}{5} \mathbb{E}[\operatorname{OPT}(2,1, \omega, \alpha)] .
\end{aligned}
$$

The bound in the above theorem is tight. For $\epsilon>0$, let $\alpha=1+\epsilon, \omega=1$ and $\mu=\epsilon$. Then, $\frac{1}{\mu}=\frac{\omega}{\alpha-1}$ and we assume that SEPT in this case processes a job of class $J^{B}$. Hence, $\mathbb{E}[\operatorname{SEPT}(2,1, \omega, \alpha)]=\frac{6}{\epsilon}$, whereas $\mathbb{E}[\operatorname{OPT}(2,1, \omega, \alpha)]=\frac{6}{\epsilon}-\frac{1}{\epsilon^{2}}\left(\frac{\epsilon}{1+\epsilon}\right)^{1+\epsilon}$, which gives

$$
\lim _{\epsilon \rightarrow 0} \frac{\mathbb{E}[\operatorname{SEPT}(2,1, \omega, \alpha)]}{\mathbb{E}[\operatorname{OPT}(2,1, \omega, \alpha)]}=\lim _{\epsilon \rightarrow 0} \frac{6}{6-\frac{1}{\epsilon}\left(\frac{\epsilon}{1+\epsilon}\right)^{1+\epsilon}}=\frac{6}{5}
$$

\subsection{Performance guarantee when in state $\left(n_{A}, 1, \omega, \alpha\right)$}

Theorem 3.

$$
\mathbb{E}\left[\operatorname{SEPT}\left(n_{A}, 1, \omega, \alpha\right)\right]<\frac{5}{4} \mathbb{E}\left[\mathrm{OPT}\left(n_{A}, 1, \omega, \alpha\right)\right]
$$


Proof. We claim that if SEPT and OPT start with different jobs, then for all $n_{A} \geq 3$

$$
\mathbb{E}\left[\mathrm{OPT}\left(n_{A}, 1, \omega, \alpha\right)\right] \geq \frac{4}{5} \mathbb{E}\left[\operatorname{SEPT}\left(n_{A}, 1, \omega, \alpha\right)\right] .
$$

Note that if SEPT and OPT start processing a job of the same class, then either they have the same sum of expected completion times, in case they both start processing the job of class $J^{B}$, or when they both start processing a job of class $J^{A}$, inductively from the claim or Theorem 2, we have that $\mathbb{E}\left[\operatorname{SEPT}\left(n_{A}, 1, \omega, \alpha\right)\right]<\frac{5}{4} \mathbb{E}\left[\mathrm{OPT}\left(n_{A}, 1, \omega, \alpha\right)\right]$.

As SEPT and OPT start processing jobs of different classes, we know by Lemma 1 that OPT will first process a job of class $J^{A}$, and the first job to be processed by SEPT is a job of class $J^{B}$. Then, it follows that $\frac{1}{\mu} \leq \frac{\omega}{\alpha-1}$. Moreover, the value of SEPT is given by

$$
\mathbb{E}\left[\operatorname{SEPT}\left(n_{A}, 1, \omega, \alpha\right)\right]=\frac{n_{A}+1}{\mu}+\frac{\omega}{\alpha-1} \sum_{i=1}^{n_{A}} i=\frac{n_{A}+1}{\mu}+\frac{n_{A}\left(n_{A}+1\right)}{2} \frac{\omega}{\alpha-1},
$$

and the optimal solution value equals

$$
\mathbb{E}\left[\mathrm{OPT}\left(n_{A}, 1, \omega, \alpha\right)\right]=\left(n_{A}+1\right) \frac{\omega}{\alpha-1}+\int_{x} \mathbb{E}\left[\mathrm{OPT}\left(n_{A}-1,1, \omega+x, \alpha+1\right)\right] f(x) \partial x .
$$

By Lemma 2, we can bound this by

$$
\begin{aligned}
\mathbb{E}\left[\operatorname{OPT}\left(n_{A}, 1, \omega, \alpha\right)\right] & >\left(n_{A}+1\right) \frac{\omega}{\alpha-1}+\frac{1}{\mu}+\frac{\omega}{\alpha-1} \sum_{i=1}^{n_{A}-1} i \\
& =\left(n_{A}+1\right) \frac{\omega}{\alpha-1}+\frac{1}{\mu}+\frac{n_{A}\left(n_{A}-1\right)}{2} \frac{\omega}{\alpha-1}
\end{aligned}
$$

Finally, since $\frac{1}{\mu} \leq \frac{\omega}{\alpha-1}$, we have

$$
\begin{aligned}
\mathbb{E}\left[\operatorname{OPT}\left(n_{A}, 1, \omega, \alpha\right)\right] & \geq \frac{4\left(n_{A}+1\right)}{5 \mu}+\left(\frac{5 n_{A}^{2}-3 n_{A}+12}{10} \frac{\omega}{\alpha-1}\right) \\
& \geq \frac{4}{5}\left(\frac{n_{A}+1}{\mu}+\frac{n_{A}\left(n_{A}+1\right)}{2} \frac{\omega}{\alpha-1}\right)=\frac{4}{5} \mathbb{E}[\operatorname{SEPT}(k+1,1, \omega, \alpha)]
\end{aligned}
$$

\section{Concluding remarks}

Theorem 1 establishes that SEPT is asymptotically optimal when the number of jobs of class $J^{A}$ tends to infinity. Intuitively the result is explained as follows. When processing and completing a job of class $J^{A}$, additional information is gained regarding the unknown parameter $\vartheta$. This information will lead to better decision making in the future. In case the expected processing time of a job of class $J^{A}$ is not minimal a trade-off arises. For small $n_{A}$, the extra information to be gained might still justify scheduling a job of class $J^{A}$. However, for large $n_{A}$, the marginal effect of this additional piece of information to our beliefs regarding $\vartheta$ is minor, as these are already based on a large number of observations. Consequently, the scheduling decisions made by SEPT almost always coincide with those made by OPT, when $n_{A}$ is large.

All obtained results are for two job classes $J^{A}$ and $J^{B}$ with $n_{B}=1$. Burnetas and Katehakis [1] study the same problem with arbitrary $n_{B} \geq 0$. From Theorem 3.4 and Remark 3.5 of their paper, it follows that in any optimal policy all jobs of class $J^{B}$ are processed consecutively. As processing a job of class $J^{B}$ reveals no information regarding $\vartheta$, SEPT processes all jobs of class 
$J^{B}$ consecutively as well. Therefore, we believe our results can be generalized without major modifications: every time we consider a single job of class $J^{B}$, a block of all jobs in class $J^{B}$ could be considered instead. Finally, we expect to be able to extend our analysis to multiple job classes as well, using results and insights from Hamada and Glazebrook [9]. They consider multiple job classes, where the processing time of a job of each class depends on a single unknown parameter.

\section{References}

[1] A.N. Burnetas and M.N. Katehakis. On sequencing two types of tasks on a single processor under incomplete information. Probability in the Engineering and Informational Sciences, $7(1): 85-119,1993$.

[2] B. C. Dean. Approximation Algorithms for Stochastic Scheduling Problems. PhD thesis, Massachusetts Institute of Technology, 2005.

[3] M.H. DeGroot. Optimal Statistical Decisions. McGraw-Hill, New York, 1970.

[4] J.C. Gittins. Bandit processes and dynamic allocation indices (with discussion). Journal of the Royal Statistical Society, Series B (Methodological), 41(2):148-177, 1979.

[5] J.C. Gittins. Multi-armed bandit allocation indices. Wiley, N.Y., 1989.

[6] J.C. Gittins and K.D. Glazebrook. On Bayesian models in stochastic scheduling. Journal of Applied Probability, 14(3):556-565, 1977.

[7] J.C. Gittins and D.M. Jones. A dynamic allocation index for the sequential design of experiments. In Progress in Statistics, pages 241-266. 1974.

[8] K.D. Glazebrook and R.W. Owen. On the value of adaptive solutions to stochastic scheduling problems. Mathematics of Operations Research, 20(1):65-89, 1995.

[9] T. Hamada and K.D. Glazebrook. A Bayesian sequential single machine scheduling problem to minimize the expected weighted sum of flowtimes of jobs with exponential processing times. Operations Research, 41(5):924-934, 1993.

[10] T. Hamada and M. Tamaki. Some results on a Bayesian sequential scheduling on two identical parallel processors. Journal of the Operations Research Society of Japan, 42(14):316-329, 1999.

[11] N. Megow, M. Uetz, and T. Vredeveld. Models and algorithms for stochastic online scheduling. Mathematics of Operations Research, 31(3):513-525, 2006.

[12] N. Megow and T. Vredeveld. Approximation results for preemptive stochastic online scheduling. In Y. Azar and T. Erlebach (eds): Proceedings of the 14th European Symposium on Algorithms (ESA 2006), pages 516-527, 2006. Lecture Notes in Computer Science; Vol. 4168.

[13] R.H. Möhring, A.S. Schulz, and M. Uetz. Approximation in stochastic scheduling:the power of lp-based priority policies. Journal of ACM, 46(6):924-942, 1999.

[14] Rolf H. Möhring, Franz Josef Radermacher, and Gideon Weiss. Stochastic scheduling problems I: General strategies. ZOR - Zeitschrift für Operations Research, 28:193-260, 1984.

[15] Rolf H. Möhring, Franz Josef Radermacher, and Gideon Weiss. Stochastic scheduling problems II: Set strategies. ZOR - Zeitschrift für Operations Research, 29(3):A65-A104, 1985.

[16] M. Pinedo. Stochastic scheduling with release dates and due dates. Operations Research, 31(3):559-572, 1983. 
[17] M. Pinedo. Off-line deterministic scheduling, stochastic scheduling, and online deterministic scheduling: A comparative overview. In J.Y.-T. Leung, editor, Handbook of scheduling: algorithms, models, and performance analysis, chapter 15. Chapman \& Hall/CRC, 2004.

[18] U. Rieder and J. Weishaupt. Customer scheduling with incomplete information. Probability in the Engineering and Informational Sciences, 9(2):269-284, 1995.

[19] M.H. Rothkopf. Scheduling with random service times. Management Science, 12(9):703-713, 1966.

[20] A.S. Schulz. New old algorithms for stochastic scheduling. In Algorithms for Optimization with Incomplete Information, 2005. Dagstuhl Seminar Proceedings, Vol. 05031.

[21] K.C. Sevcik. Scheduling for minimum total loss using service time distributions. Journal of the ACM, 21(1):66-75, 1974.

[22] M. Skutella and M. Uetz. Stochastic machine scheduling with precedence constraints. SIAM Journal on Computing, 34(4):788-802, 2005.

[23] G. Weiss. Approximation results in parallel machines stochastic scheduling. Annals of Operations Research, 26(1):195-242, 1990.

[24] G. Weiss. Turnpike optimality of Smith's rule in parallel machines stochastic scheduling. Mathematics of Operations Research, 17(2):255-270, 1992.

[25] G. Weiss. On almost optimal priority rules for preemptive scheduling of stochastic jobs on parallel machines. Advances in Applied Probability, 27:827-845, 1995. 